\title{
Effect of Pressure on Magnetic Properties of $\left(\mathrm{NH}_{3} \mathrm{OH}\right)_{2} \mathrm{CoF}_{4}$ Fluoro-Metal Complex
}

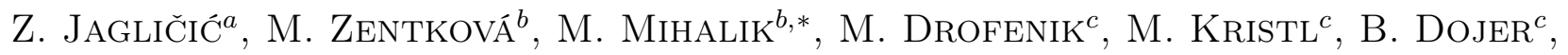

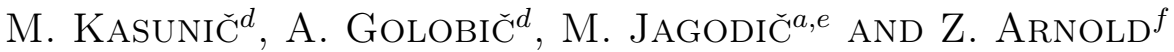 \\ ${ }^{a}$ Institute of Mathematics, Physics and Mechanics and Faculty of Civil and Geodetic Engineering \\ Jadranska 19, SI-1000 Ljubljana, Slovenia \\ ${ }^{b}$ Institute of Experimental Physics, SAS, Watsonova 47, 04001 Košice, Slovakia \\ ${ }^{c}$ Faculty of Chemistry and Chemical Engineering, University of Maribor, Smetanova 17, SI-2000 Slovenia \\ ${ }^{d}$ Faculty of Chemistry and Chemical Technology, University of Ljubljana, Aškerčeva 5, SI-1000 Slovenia \\ ${ }^{e}$ EN-FIST Centre of Excellence, Dunajska 156, 1000 Ljubljana, Slovenia \\ ${ }^{f}$ Institute of Physics ASCR, v.v.i., Na Slovance 2, 18221 Prague 8, Czech Republic
}

\begin{abstract}
Effect of pressure on magnetic properties of a bulk fluoro-metal complex $\left(\mathrm{NH}_{3} \mathrm{OH}\right)_{2} \mathrm{MF}_{4}$ was studied. Magnetization measurements suggest that a ferromagnetic transition at $T_{\mathrm{C} 1}=47 \mathrm{~K}$ is followed by a ferrimagnetic one at $T_{\mathrm{C} 2}=3 \mathrm{~K}$. Both transition temperatures are pressure dependent with the pressure coefficients $\mathrm{d} T_{\mathrm{C} 1} / \mathrm{d} p=-2.6 \mathrm{~K} / \mathrm{GPa}$ and $\mathrm{d} T_{\mathrm{C} 2} / \mathrm{d} p=0.26 \mathrm{~K} / \mathrm{GPa}$. The opposite sign of the coefficients is an additional indication of a different nature (ferromagnetic/antiferromagnetic) of these two transitions. The effect of pressure on low field magnetization and exchange bias phenomena is small but still visible.
\end{abstract}

PACS numbers: 75.47.Lx, 75.30.Cr, 75.30.Kz

\section{Introduction}

Hydroxylammonium fluorometalates with a general formula $\left(\mathrm{NH}_{3} \mathrm{OH}\right)_{x} \mathrm{MeF}_{y}$ are interesting for the study of hydrogen bonds, since they include all three elements that are capable to form strong hydrogen bonds $(\mathrm{O}, \mathrm{N}$ and F) [1]. Layered fluoro-metal complexes have recently attracted attention due to their structural similarity to layered copper oxides, like e.g. $\mathrm{La}_{2} \mathrm{CuO}_{4}$, which is important precursor of the high- $T_{\mathrm{c}}$ oxocuprate superconductors. A new layered fluoro-metal complex with rich magnetic properties: two magnetic transitions and exchange bias in bulk sample below $10 \mathrm{~K}$ was synthesized recently [1]. Exchange bias is a phenomenona where the magnetization of an antiferromagnetic layer causes a shift in the $M(H)$ curve of a ferromagnetic layer. The phenomenon is very important in magnetic recording. Usually it occurs in bilayer or multilayer systems and is very rarely observed in bulk materials. In our paper we focus on the effect of high pressure on magnetic properties of $\left(\mathrm{NH}_{3} \mathrm{OH}\right)_{2} \mathrm{CoF}_{4}$ layered fluoro-metal complex.

Layered hydroxylammonium fluorometalate $\left(\mathrm{NH}_{3} \mathrm{OH}\right)_{2} \mathrm{CoF}_{4}$ was prepared by the reaction of solid $\mathrm{NH}_{3} \mathrm{OHF}$ and the aqueous solution of copper or cobalt in HF. The compound crystallizes in monoclinic, $P 2_{1} / c$, unit cell with parameters: $a=8.1764(3) \AA$,

\footnotetext{
* corresponding author; e-mail: mihalik@saske.sk
}

$b=5.8571(2) \AA, c=5.6662(2) \AA, \beta=94.675(3)^{\circ}[1]$. All magnetization measurements were performed by a SQUID magnetometer MPMS XL-7 in temperature range between $1.8 \mathrm{~K}$ and $300 \mathrm{~K}$ and in magnetic fields with induction up to $7 \mathrm{~T}$. The sample was placed in a pressure cell during all magnetic measurements. A piston cylinder type of the $\mathrm{CuBe}$ pressure cell was filled up with the mixture of mineral oils serving as hydrostatic pressure transmitting medium and operating up to $1.2 \mathrm{GPa}[2]$. Actual pressure was determined in situ from the pressure dependence of the superconducting transition temperature $T_{\mathrm{c}}(p)$ of an inserted high-purity lead sample. The pressure CuBe cell is permanently present in all detecting coils of the SQUID magnetometer and hence, it practically does not contribute to overall magnetization.

Figure 1 shows temperature dependences of magnetization which were measured in zero field cooled (ZFC), field cooled (FC) regimes and at different hydrostatic pressures. The magnetization slowly increases from room temperature down to $50 \mathrm{~K}$ then the magnetization steeply increases and the compound undergoes paramagnetic to ferromagnetic $(\mathrm{FM})$ transition at $T_{\mathrm{C} 1}=47 \mathrm{~K}$. The Curie temperature $T_{\mathrm{C} 1}$ was determined as a minimum of the $\mathrm{d} \mu / \mathrm{d} T(T)$ dependences. The FM character of coupling between magnetic moments was demonstrated in [1]. Below the bifurcation temperature $T_{\mathrm{b}}=$ $35 \mathrm{~K}$, the ZFC and FC curves show hysteretic behaviour which is not as pronounced as it was shown in [1] but still 


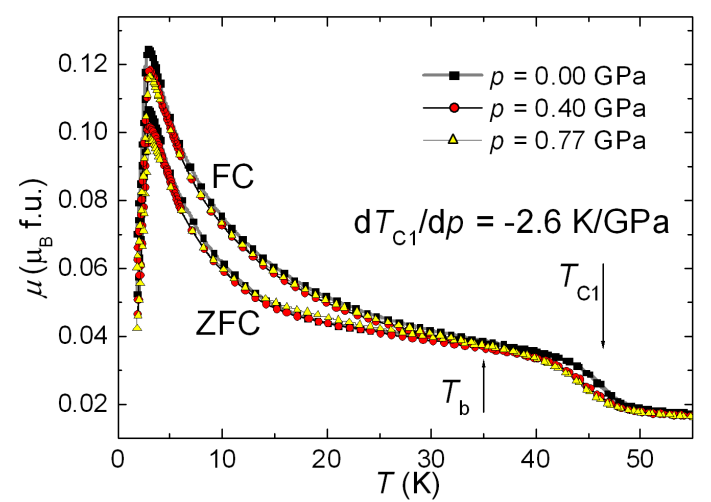

Fig. 1. Temperature dependences of magnetization were measured in magnetic field with induction of $0.8 \mathrm{~T}$ and in ZFC and FC regimes at different hydrostatic pressures.

is remarkable. The large maximum at about $T_{\mathrm{C} 2}=3 \mathrm{~K}$ is associated with another magnetic transition. Such a maximum is a typical feature of systems with antiferromagnetic (AF) ordering. Microscopic origin of magnetic ordering is still not clear, one possibility - ferrimagnetic ordering - was proposed in [1]. Magnetic ordering in this compound can be more complex and another explanation is that due to the layered structure of the compound both FM and AFM orderings are present and contribute to overall magnetization. Applied hydrostatic pressure reduces the Curie temperature nearly linearly with the pressure coefficient $\mathrm{d} T_{\mathrm{C} 1} / \mathrm{d} p=-2.6 \mathrm{~K} / \mathrm{GPa}$. The bifurcation temperature $T_{\mathrm{b}}$ is not affected by pressure.

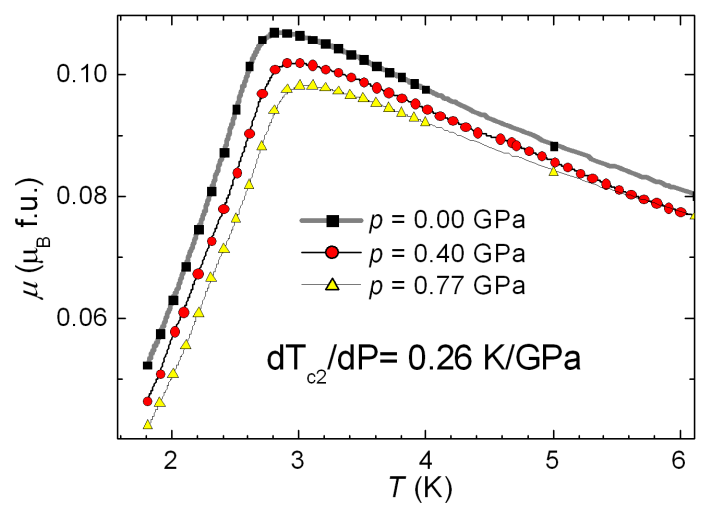

Fig. 2. Pressure effect on the magnetic phase transition at $T_{\mathrm{C} 2}$.

The effect of hydrostatic pressure on the low temperature maximum on $M(T)$ curve is shown in Fig. 2. Pressure reduces the maximum and shifts it to higher temperature which indicates the increase of $T_{\mathrm{C} 2}$ with pressure coefficient $\mathrm{d} T_{\mathrm{C} 2} / \mathrm{d} p=0.26 \mathrm{~K} / \mathrm{GPa}$. The effect of pressure on $T_{\mathrm{C} 1}$ is opposite as on $T_{\mathrm{C} 2}$ which can again indicate different coupling between magnetic moments: ferromagnetic (FM) and antiferromagnetic (AFM). The field dependence of magnetization measured at $1.8 \mathrm{~K}$ displays S-shape, it has a tendency to saturate only slowly reaching the value $0.73 \mu_{\mathrm{B}} /$ f.u. at $7 \mathrm{~T}$. The applied pressure does not affect high field magnetization. The low field magnetization is shown in Fig. 3. The asymmetry of hysteretic loop points out to exchange bias phenomena. The asymmetry is remarkable from value of coercive fields; there is no asymmetry in remanent magnetization. Magnetization reaches zero value for magnetic fields with induction of $0.3029 \mathrm{~T}$ and $0.2109 \mathrm{~T}$ for pressure $0.4 \mathrm{GPa}$ and $0.3519 \mathrm{~T}$ and $0.2912 \mathrm{~T}$ for pressure 0.77 GPa. The low field magnetization is found to be sensitive to external parameters like pressure coercive field increases with pressure. It seems that exchange bias phenomenon was a little bit suppressed by pressure.

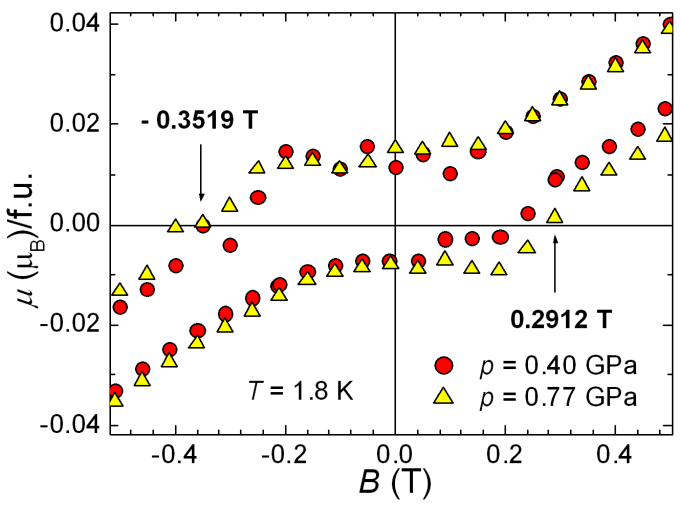

Fig. 3. Low field magnetization dependences of $\left(\mathrm{NH}_{3} \mathrm{OH}\right)_{2} \mathrm{CoF}_{4}$ compound were measured at $1.8 \mathrm{~K}$ and at different pressures.

Our study revealed that both magnetic transitions are sensitive on pressure but the effect of pressure is different on both magnetic transitions. The upper critical temperature $T_{\mathrm{C} 1}$ decreases and the lower critical temperature $T_{\mathrm{C} 2}$ increases with pressure. The low field magnetization and exchange bias phenomena are affected by pressure, the value of coercive force is higher under pressure. The microscopic magnetic structure of the compound is not known at present but there are indications that layered structure of the compound is responsible for a complex magnetic behaviour. We expect that FM coupling is dominant below $T_{\mathrm{C} 1}$ and AFM coupling is characteristic feature of the system below $T_{\mathrm{C} 2}$.

\section{Acknowledgments}

This work was supported by the projects VEGA 2/ 0057/10 and SK-SI-0032-08.

\section{References}

[1] M. Kristl, B. Dojer, M. Kasunič, A. Golobič, Z. Jagličić, M. Drofenik, J. Fluorine Chem. 131, 907 (2010).

[2] J. Kamarád, Z. Machátová, Z. Arnold, Rev. Sci. Instrum. 75, 5022 (2004). 